\title{
Surgical risk and technical notes of laparoscopic anterior resection in the elderly: lessons from an early experience Giovanni D Tebala*1, Ida Camperchioli ${ }^{2}$, Paolo Innocenti ${ }^{1}$, Nicola Di Lorenzo $^{2}$, Pierpaolo Sileri ${ }^{2}$ and Achille L Gaspari ${ }^{2}$
}

\author{
Address: ${ }^{1}$ Digestive Surgery Unit, Department of Surgery, Aurelia Hospital, Rome 0039, Italy and ${ }^{2}$ Department of Surgery, Policlinico Tor Vergata, \\ Rome 0039, Italy \\ * Corresponding author
}

\author{
from XXI Annual Meeting of The Italian Society of Geriatric Surgery \\ Terni, Italy. 4-6 December 2008 \\ Published: I April 2009 \\ BMC Geriatrics 2009, 9(SuppI I):A62 doi:10.1 186/I47I-23I8-9-SI-A62
}

This abstract is available from: http://www.biomedcentral.com/I47I-23I8/9/SI/A62

(c) 2009 Tebala et al; licensee BioMed Central Ltd.

\section{Background}

The many advantages and the few limits of laparoscopic surgery have already been demonstrated, but its role in colorectal surgical oncology are all but cleared. However, recent series show that short- and long-term results are the same of traditional surgery. Some concern still remains on the safety of laparoscopic oncologic surgery in aged patients with their expected comorbidity. But elderly patients are those in whom a mini-invasive approach could be most beneficial.

\section{Materials and methods}

We retrospectively evaluated our initial experience with aged patients who underwent laparoscopic anterior rectal resection, trying to answer to the following questions: what is the surgical risk? What are the technical and strategic tips? Seventeen patients aged $\geq 65$ years underwent surgical treatment for sigmoid or rectal cancer up to T2 (11 pts), chronic diverticular disease with substenosis (5 pts), benign disease not amenable to endoscopic treatment ( 1 pt). Contraindications were: ischemic heart disease, severe anemia, chronic respiratory distress and coagulopathy. Our technique entails the routine mobilization of the splenic flexure, the section of the inferior mesenteric artery at its origin and of the inferior mesenteric vein at the Treitz level and the execution of a colorectal anastomosis well below the peritoneal pelvic reflexion, paying attention to the good vascularization and the total absence of tension.

\section{Results}

From the presented series we excluded the first "learning" cases in which the operation was always converted after an early laparoscopic phase. Fourteen operations were completed by laparoscopy. Conversion was necessary due to intraoperative bleeding ( $1 \mathrm{pt})$ and impossibility to manipulate and mobilize the rectum ( 2 pts, a bulging rectal cancer and a huge diverticular mass). Mean operative time was $186 \pm 29$ minutes. The patient is mobilized in postoperative day (p.o.d.) 1; bowel movements are present up to p.o.d. 2 and oral intake is started on p.o.d. 5. No severe complications were registered but an incisional hernia on a McBurney minilaparotomy, which was treated by laparoscopy 3 months later.

\section{Conclusion}

This initial experience allows us to guess that laparoscopic approach is a safe and efficient technique also in the elderly, if attention is paid to patient selection and if the classical surgical rules are followed. In our opinion, it's extremely important that the technique is someway standardized and the usual manoeuvres are always repeated independently of the disease and the anatomy. 